\title{
The MAMI Care Pathway Package: A resource to support the management of small and nutritionally at-risk infants under six months of age and their mothers (MAMI)
}

\author{
Kelsey Grey ${ }^{1}$, Eilise Brennan ${ }^{1}$, Marko \\ Kerac², Marie McGrath' \\ 1. Emergency Nutrition Network, \\ Oxford, UK \\ 2. London School of Hygiene and \\ Tropical Medicine, London, UK
}

Correspondence:

Kelsey Grey

kelsey@ennonline.net

Submitted: June 2021

Accepted: July 2021

Published: August 2021
Citation:

Grey et al. The MAMI Care Pathway Package: A resource to support the management of small and nutritionally at-risk infants under six months of age and their mothers (MAMI). South Sudan Medical Journal 2021;14(3):94-97 (c) 2021 The Author (s) License: This is an open access article under CC BY-NC-ND DOI: https://dx.doi.org/10.4314/ssmj.v14i3.6

\section{Abstract}

Globally, millions of infants under six months $(\mathrm{u} 6 \mathrm{~m})$ are small and nutritionally at-risk, but many do not get the care they need to survive and thrive. Although the 2013 World Health Organisation (WHO) guidelines for severe malnutrition management recommend outpatient care for clinically stable infants $\mathrm{u} 6 \mathrm{~m}$, most national guidelines still recommend inpatient care for all infants u6m. To help put the WHO recommendations into action, the MAMI Global Network has developed the MAMI Care Pathway Package a resource to facilitate the screening, assessment, and management of small and nutritionally at-risk infants $\mathrm{u} 6 \mathrm{~m}$ and their mothers. The Package uses an integrated care pathway approach and is designed to embed within and support Integrated Management of Childhood Illness (IMCI). By improving continuity of care and facilitating patient management, the MAMI Care Pathway Package aims to help health workers improve outcomes for infants and mothers worldwide while also simplifying their care.

Keywords: MAMI, care pathway, at-risk infants, wasting

\section{Introduction}

\section{The burden of small and nutritionally at-risk infants under six months}

Nearly half of all child deaths are due to undernutrition and infants $\mathrm{u} 6 \mathrm{~m}$ are the most vulnerable. ${ }^{[1,2]}$ In low- and middle-income countries (LMICs), about 1 in 5 infants $\mathrm{u} 6 \mathrm{~m}$ is small and nutritionally at-risk. ${ }^{[3]}$ They may be born small and at risk (low birth weight: small for gestational age and/or pre-term infants) or may develop nutritional risk after birth (growth faltering, wasting, stunting, and/or underweight infants).

These infants are at higher risk of sickness, death, and poor development. Globally there are an estimated 8.5 million infants $\mathrm{u} 6 \mathrm{~m}$ who are wasted and an even greater number who are small and nutritionally at-risk. ${ }^{[4]}$ In South Sudan, about $23 \%$ of children under five years are wasted. ${ }^{[5]}$

The critical gap in care for small and nutritionally at-risk infants $u 6 \mathrm{~m}$ and their mothers

Despite the millions of infants and their mothers in this situation worldwide, many do not get the care that they need to survive and thrive. This is because there is a serious gap in care for these infants and their mothers, especially in community services and after the new-born period. Neonatal services often have limited involvement after six weeks of age, while services for the management of wasting are designed for children from age six months. Routine infant and young child feeding (IYCF) counselling is not sufficient to meet their more complex needs. Most current national malnutrition guidelines make recommendations 
for inpatient care for this vulnerable group, but since this has high costs and opportunity costs, many families struggle to access this care. ${ }^{[6,7]}$ Many of these infants are clinically stable and could be managed in outpatient care that is more accessible for families and a more appropriate setting for the type of support they need. However, despite being recommended in the $2013 \mathrm{WHO}$ guidelines for the management of severe malnutrition, outpatient care for infants $\mathrm{u} 6 \mathrm{~m}$ is rarely available; countries need more guidance on how to put the recommendations into practice, more evidence of what works in their context, and how and what are the resource needs.

\section{The MAMI Global Network}

In 2010, the MAMI Global Network (formerly the MAMI Special Interest Group) was formed by the Emergency Nutrition Network (ENN) to bring attention to the burden and care gap in the management of small and nutritionally at-risk infants $\mathrm{u} 6 \mathrm{~m}$ and their mothers (MAMI). The MAMI Global Network involves local, national, and international collaborators working together to improve policy, evidence, and practice. The vision of the MAMI Global Network is that "every small and nutritionally at-risk infant $\mathrm{u} 6 \mathrm{~m}$ and their mother is supported to survive and thrive."

\section{The MAMI Care Pathway Package}

To help put this vision into action and to support health workers to manage cases that were presenting to their health facilities and programmes, the MAMI Global Network developed the first version of the C-MAMI Tool in 2015. The most recent version, redesigned as the MAMI Care Pathway Package, was released in May 2021 (Version 3). This update was informed by the latest evidence and experiences of putting previous versions into practice. The MAMI Care Pathway Package is a package of resources that was co-created by members of the MAMI Global Network, co-led by ENN and the London School of Hygiene and Tropical Medicine (LSHTM). It provides practical, field-orientated guidance on how to screen, assess, and support small and nutritionally atrisk infants $\mathrm{u} 6 \mathrm{~m}$ and their mothers. Support materials help practitioners put this into practice. The MAMI Care Pathway Package will be formally tested in a randomised controlled trial in Ethiopia (2021-2024).

\section{Guiding principles of the MAMI Care Pathway}

- The MAMI Care Pathway always considers the infant and mother together because infant wellbeing is inextricably linked to maternal wellbeing.

- The MAMI Care Pathway is about 'bridging', linking with, and using existing systems and services. Since there are many possible underlying causes of growth faltering/anthropometric deficit in this age group, effective management is not just about nutrition/breastfeeding support. It also involves health, mental health, and social interventions to provide more holistic care for atrisk infants $\mathrm{u} 6 \mathrm{~m}$ and their mothers.

- It helps put the 2013 WHO guidelines for the management of severe malnutrition into practice and is designed to embed within IMCI, an established approach to child health implemented by families, communities, and health facilities.

- The MAMI Care Pathway is based on an integrated care pathway approach that considers the processes, resources, and participation needed to support infants and mothers from initial screening through to exit from care.

- While in some settings the MAMI Care Pathway can be used directly or 'off-the-shelf, it will often require context-specific adaptation to fit with local needs and resources that already exist.

- The MAMI Care Pathway is relevant across development and humanitarian settings and different contexts. It also links treatment and prevention: treating a small and nutritionally atrisk infant $\mathrm{u} 6 \mathrm{~m}$ in the short-term aims to prevent that individual from developing even more serious nutritional disorders such as stunting and wasting in later childhood.

\section{How does the MAMI Care Pathway work in practice?}

The MAMI Care Pathway promotes identification, assessment, and support of infants $\mathrm{u} 6 \mathrm{~m}$ and their mothers at every community and health service contact point. There are many opportunities after birth to identify small and nutritionally at-risk infants and offer support. For example, infants with low birthweights can be identified for community follow up, while infants attending for vaccinations come into contact with health services several times before six months of age. It is important to take these opportunities to identify at-risk infants $\mathrm{u} 6 \mathrm{~m}$ and mothers, to provide or refer for appropriate support, and to monitor their progress.

The MAMI Care Pathway has three key stages: rapid screening, assessment, and support and management. Each stage is supported by user guides and forms for frontline health workers (Figure 1). These materials can be used directly or may inform adaptations to existing materials and resources already used by health workers.

\section{Contents of the MAMI Care Pathway Package}

There are three core materials in the MAMI Care Pathway Package :

1. User guides: these serve as training materials/references 


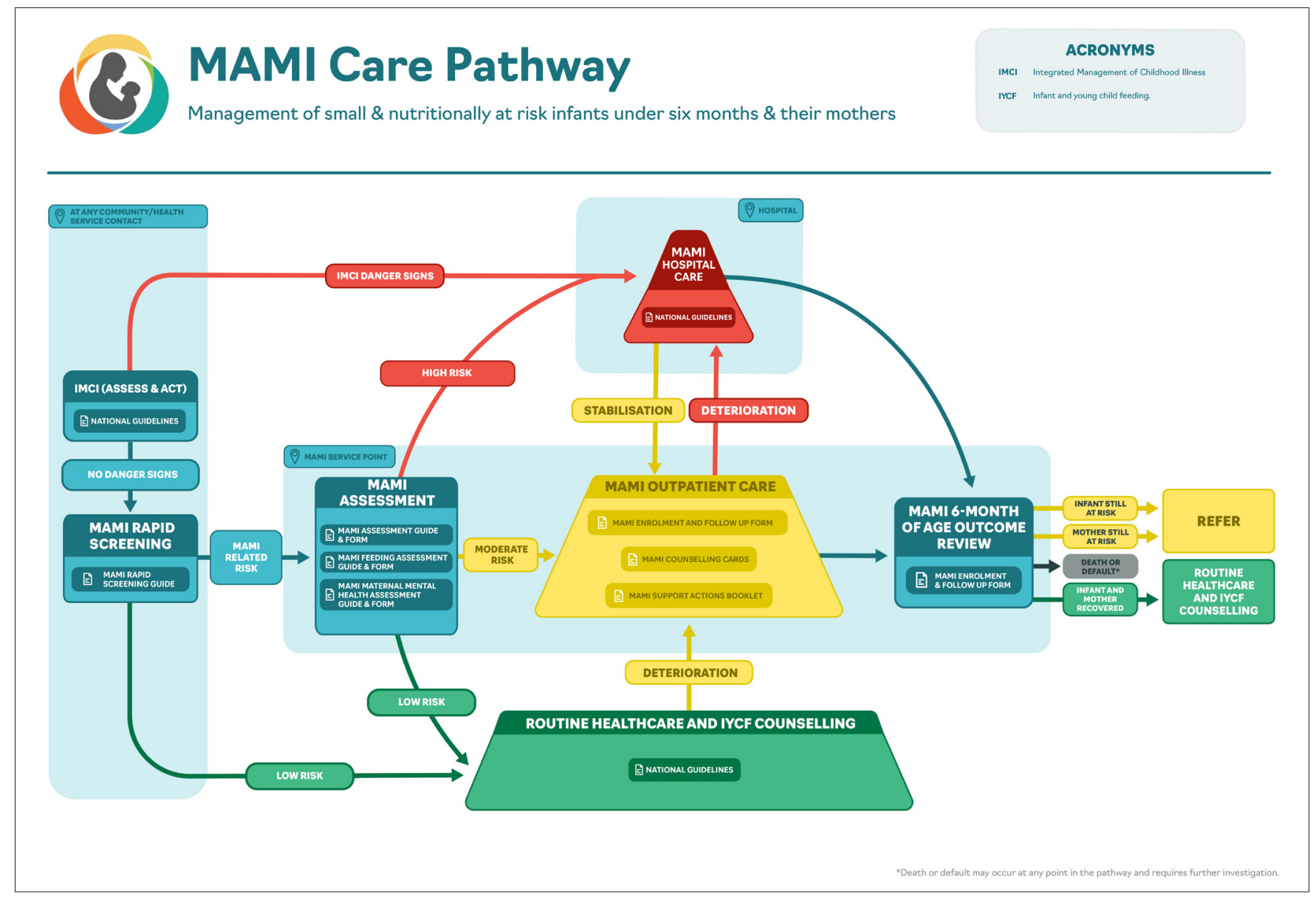

Figure 1. An overview of the MAMI Care Pathway Package. Source: MAMI Care Pathway Package.

and are formatted in the style of IMCI. For example, see The MAMI Rapid Screening guide formatted in IMCI style at https://www.ennonline.net/attachments/3890/ MAMI-Rapid-Screening-Guide.pdf.

2. Forms: to help healthcare workers gather key patient data and help them manage patients. For example, see The MAMI Assessment Form at https://www.ennonline. net/attachments/3896/MAMI-Assessment-Form.pdf.

3. Counselling cards and support actions booklet: to facilitate assessment and management by frontline health workers. These are largely based on the UNICEF IYCF counselling cards that draw upon WHO resources and experts, with MAMI-specific additions. Again, these may be adapted or used to complement existing materials.

https://www.ennonline.net/attachments/3905/MAMICounselling-Cards-(standard-version-horizontal).pdf

\section{Benefits of the MAMI Care Pathway}

The MAMI Care Pathway benefits multiple stakeholders at multiple levels.

First and foremost, it benefits small and nutritionally at-risk infants $\mathrm{u} 6 \mathrm{~m}$ and their mothers by improving the quality and continuity of care that they receive, helping them to survive and thrive. Improving the health and nutrition of infants $\mathrm{u} 6 \mathrm{~m}$ has short- and long-term benefits for these infants, their families, and for wider society.

It also benefits frontline health care workers by:

- Increasing job satisfaction by offering easy-tofollow guides and solutions to deal with complex problems.

- Helping ensure that serious underlying conditions are not missed - a situation that can create stress for health workers.

- Reducing patient workload by allowing health workers to identify and focus on the infants and mothers with the highest needs and intervening early so as to prevent more complex, timeconsuming problems later on.

- Recognising that referral services can be limited or delayed and that health workers need a simple 'how to' guide for immediate support.

For service managers and trainers, the MAMI Care Pathway Package provides a ready-made suite of materials 
and resources to help implement improved care for infants $\mathrm{u} 6 \mathrm{~m}$ and their mothers. It also makes it easier to audit services and monitor performance.

Policy makers and researchers also benefit - the package translates existing policies for front-line field use; helps generate evidence that is needed for future scale-up; and bridges existing services, especially across health and nutrition.

\section{Conclusion}

The MAMI Care Pathway is an integrated care pathway that aims to improve the care of small and nutritionally at-risk infants $\mathrm{u} 6 \mathrm{~m}$ and their mothers. It provides implementation guidance and materials to help put this care into practice. It sometimes requires adaptation to different contexts. To help fill the gap in care for these infants and their mothers, it is critical to document and share experiences of adapting and implementing the MAMI Care Pathway Package in different contexts. This will help build the evidence of what works and how and will be used to develop future versions.

- The MAMI Care Pathway Package is available online at https://www.ennonline.net/ mamicarepathway.

- Orientation videos on the MAMI Care Pathway Package are available on the MAMI Global Network Youtube channel. See Part 1 MAMI Care Pathway Package Overview presentation (20 mins); Part 2 MAMI Care Pathway Package Overview presentation (10 mins) and MAMI Care Pathway Package webinar recording (1 hour 30 mins)

- Share your experiences and become part of global action on MAMI by joining the MAMI Global Network. Contact the MAMI Global Network Coordinator, mami@ennonline.net

- Ask urgent questions on the dedicated MAMI page of ENN's online technical forum, en-net, https://www.en-net.org/forum/19.aspx.

The authors declare no conflict of interest.

The development of the MAMI Care Pathway Package (2021) was funded by the Eleanor Crook Foundation, Irish Aid, and the Waterloo Foundation, and coordinated by the Emergency Nutrition Network. We acknowledge the enormous support of the MAMI Global Network members who contributed to the development of the MAMI Care Pathway Package.

\section{References}

1. Black RE, Victora CG, Walker SP, Bhutta ZA, Christian P, de Onis M, et al. Maternal and child undernutrition and overweight in lowincome and middle-income countries. Lancet. 2013;382(9890):427-51. DOI: https://doi. org/10.1016/S0140-6736(13)60937-X.

2. Grijalva-Eternod CS, Kerac M, McGrath M, Wilkinson C, Hirsch JC, Delchevalerie P, et al. Admission profile and discharge outcomes for infants aged less than 6 months admitted to inpatient therapeutic care in 10 countries. A secondary data analysis. Matern Child Nutr. 2017;13(3). DOI: https://onlinelibrary.wiley. com/doi/epdf/10.1111/mcn.12345.

3. Kerac et al. 2019. Analysis presented at World Health Organization expert consultation, Geneva.

4. Kerac M, Blencowe $\mathrm{H}$, Grijalva-Eternod C, McGrath M, Shoham J, Cole TJ, et al. Prevalence of wasting among under 6-month-old infants in developing countries and implications of new case definitions using WHO growth standards: a secondary data analysis. Archives of disease in childhood. 2011;96(11). DOI: 10.1136/ adc.2010.191882.

5. Global Nutrition Report. Action on equity to end malnutrition. Bristol, UK: Development Initiatives; 2020. https:/globalnutritionreport. org/reports/2020-global-nutrition-report/

6. Kerac M, Angood C, McGrath M, Lelijveld N, Trehan I, Manary M. Towards rollout of new who guidelines for improved management of severe acute malnutrition in infants aged $<6$ months: an agree appraisal of national guidelines. Nutrition \& Growth Conference; 3rd March 2017; Amsterdam, The Netherlands, 2017. DOI: $10.1111 / \mathrm{mcn} .12642$.

7. Munirul Islam $\mathrm{M}$, Arafat $\mathrm{Y}$, Connell $\mathrm{N}$, Mothabbir G, McGrath M, Berkley JA, et al. Severe malnutrition in infants aged $<6$ months - outcomes and risk factors in Bangladesh: A prospective cohort study. Matern Child Nutr. 2019;15(1):e12642. DOI: 10.1111/mcn.12642. 